\title{
Galactic Archaeology with the Subaru Prime Focus Spectrograph
}

\author{
Masashi Chiba ${ }^{1}$, Judith Cohen ${ }^{2}$ and Rosemary F. G. Wyse ${ }^{3}$ \\ ${ }^{1}$ Astronomical Institute, Tohoku University, Sendai, Japan \\ email: chiba@astr.tohoku.ac.jp \\ ${ }^{2}$ Caltech, Pasadena, CA, USA \\ email: jlc@astro.caltech.edu \\ ${ }^{3}$ Johns Hopkins University, Baltimore, MD, USA \\ email: wyse@jhu.edu
}

\begin{abstract}
We present an overview of our Galactic Archaeology (GA) survey program with the Prime Focus Spectrograph (PFS) for Subaru. Following successful design reviews, the instrument is now under construction with first light anticipated in 2018. Main characteristics of PFS and the science goals in our PFS/GA program are described.
\end{abstract}

Keywords. Galactic Archaeology, Subaru/PFS

\section{What is PFS?}

PFS is a massively-multiplexed, fiber-fed optical and near-infrared 3-arm spectrograph, to be mounted on Subaru (Fig.1). Main characteristics of PFS are summarized as follows.

- Funding started in 2010 (PI: H. Murayama).

- Developed based on international collaboration: Kavli IPMU (U. of Tokyo), NAOJ, ASIAA, LNA, Caltech/JPL, LAM, Princeton, JHU, IAG/Uni de Sao Paulo and MPA.

- Field of view: 1.4 degree in diameter.

- Number of fibers: 2400.

- Spectrograph: 3-arm design to cover 380-1260 nm simultaneously, i.e., blue (3800$6700 \AA)$, red (6500-10000 $\AA$ ) and IR arms (9700-13000 $)$.

- Resolution: LR mode $(R=2,000-3,000)$ and MR mode $(R=5,000)$

- First light: tentatively scheduled in 2018.

\section{GA survey}

GA is one of three main science components of the Subaru Strategic Program for PFS (see Takada et al. 2014 for details). We expect to have 300 to 360 nights for the entire PFS program, of which about 100 nights are expected to go to the GA program. With PFS, we will measure radial velocities and chemical abundances of stars in the Milky Way and M31 to infer the past assembly histories of these galaxies and the structure of their dark matter halos. Science requirements are summarized in Table 1. Data will be secured for numerous stars in the Galactic thick-disk, halo and tidal streams as faint as $V=22$ mag with a low-resolution mode with $R=2,000$ to 3,000, including stars with $V<20$ mag to complement the goals of the Gaia mission. A medium-resolution mode with $R=5,000$ to be implemented in the red arm will allow the measurement of multiple alpha-element abundances (Left panel of Fig.2) and more precise velocities for Galactic stars, elucidating the detailed chemo-dynamical structure and evolution of each of the main stellar components of the Milky Way Galaxy and of its dwarf spheroidal galaxies (Right panel of Fig. 2). The M31 halo campaign will target red giant branch stars with 


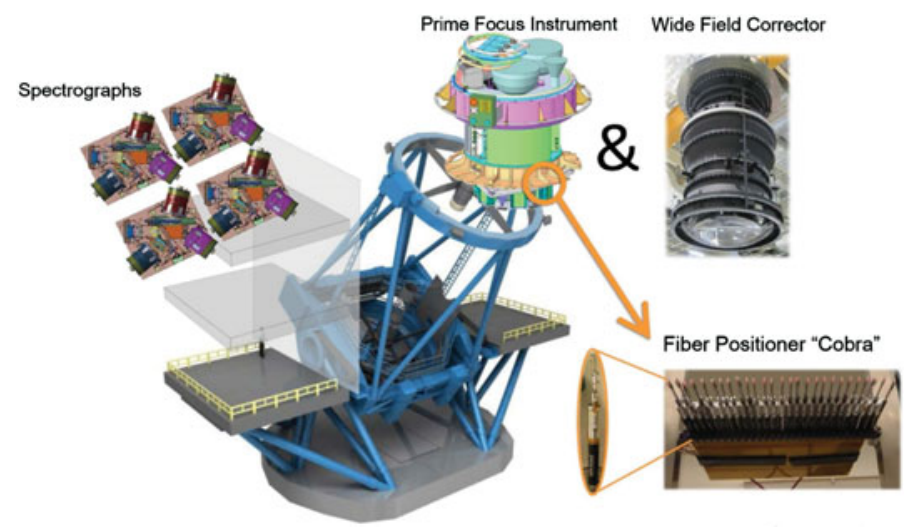

Figure 1. An overview of the baseline design of Subaru/PFS (taken from http://pfs.ipmu.jp/instrumentation.html).
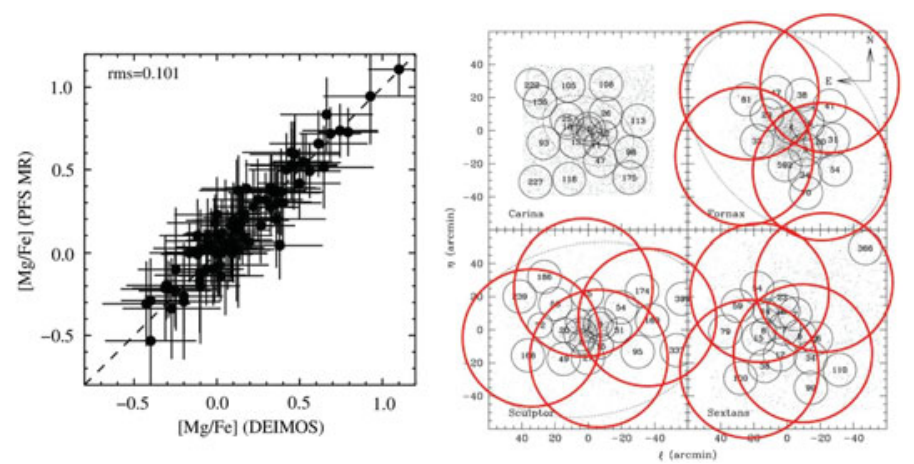

Figure 2. Left: Comparison of $[\mathrm{Mg} / \mathrm{Fe}]$ derived from PFS-MR spectra to those of the DEIMOS data. Right: Proposed PFS pointings (solid circles) for the wide-field velocity measurements of some Galactic dSphs (Fornax, Sculptor and Sextans) reaching their tidal radii (dotted lines) (adapted from Walker et al. 2009).

Table 1. Summary of Galactic Archaeology Requirements for PFS

\begin{tabular}{ll}
\hline Mode & Requirements \& Comments \\
\hline LR & For the Milky Way stars $(V<22)$ and M31 halo $(21.5<V<22.5)$ \\
$(R=2000-3000)$ & Velocity precision of $5-10 \mathrm{~km} \mathrm{~s}^{-1}$ \\
& {$[\mathrm{Fe} / \mathrm{H}]$ to $\sim 0.2$ dex } \\
& $\lambda=3800 \AA$ to $1 \mu \mathrm{m}$ incl. Ca II HK, Ca I, Mgb/MgH, CaT \\
\hline MR & For the Milky Way stars $(V<19$ in bright and $V<21$ in grey/dark time $)$ \\
$(R=5000)$ & Velocity precision of $3 \mathrm{~km} \mathrm{~s}{ }^{-1}$ \\
& {$[$ Fe $/ \mathrm{H}]$ to 0.15 dex, $[\mathrm{X} / \mathrm{Fe}](\mathrm{X}=\mathrm{Mg}, \mathrm{Si}, \mathrm{Ca}, \mathrm{Ti})$ to $\sim 0.2$ dex } \\
& $\lambda=7100$ to $8850 \AA$ incl. CaT and $\alpha$-element lines \\
\hline
\end{tabular}

$21.5<V<22.5 \mathrm{mag}$, obtaining radial velocities and spectroscopic metallicities over an unprecedented large area of its stellar halo. In synergy with these planned PFS survey, the coordinated imaging surveys with Hyper Suprime Cam are currently going on.

\section{References}

Takada, M. et al. 2014, PASJ, 66, 1

Walker, M. G. et al. 2009, AJ, 137, 3100 